\title{
Optimization of HF waves energy source for thermal treatment of building materials
}

\author{
Irina Vorotyntseva \\ Moscow State University of Civil Engineering, Yaroslavskoe shosse, 26, Moscow, 129337, Russia
}

\begin{abstract}
Building materials processing with the help of HF waves demonstrates a great number of perspective advantages as compared to traditional heating methods. In order to upgrade the technology of HF wave heating there exist a need to optimize the HF waves sources that enable us to consider some characteristic features of the process to a greater extend. To solve the task of optimizing a HF wave energy source we use the methods of the optimal control theory. The optimization has been carried out based on the gradient method. As a result we have found some optimal functional dependencies that describe the laws strength change of an electrostatic and a high-frequency field. Established managements help considerably enhance the efficiency of the energy exchange. The calculations we have carried out show that the chosen method enables an efficient optimization of a HF wave energy source with different restrictions of the governing function.
\end{abstract}

\section{Introduction}

Using the energy of HF (high-frequency) electromagnetic field for the purpose of thermal treatment of dielectric materials enables intensive, wasteless, energy-saving and ecologically clean technologies $[1,2]$. The development of microwave technologies foresees the production of new high-strength construction and building materials made of fiberglass reinforced plastic, composites and polymers.

While studying modern HF wave technologies for thermal treatment of building materials great attention is being paid to the search for new technical solutions that allow some given temperature distribution within the whole volume of the material in work $[3,4]$. This task is particularly important for the thermal treatment of considerably thick materials (3-6 wave lengths of the HF wave energy source) that also have a low coefficient of thermal conductivity. Exactly these conditions and restrictions are being displayed while producing building materials. This fact is determined by the conditions of the technological process for the realization of the needed solidity characteristics of the manufactured articles with regard to the fullness of the hydration process (concrete, foam concrete with different fillings), polymerization (goods made of fiberglass and polymers, composites), as well as absence of any internal mechanical tensions.

HF wave thermal treatment pays an important role in producing heat-insulating building materials due to high inefficiency of the existing convection heating methods which is responsible for the low coefficient of thermal conductivity of heat-insulating materials [5]. 
The development of new HF wave devices that enable the given temperature distribution in the volume of the material in work as well as the optimization of existing HF wave energy sources is a task of current importance in the field of building materials production.

\section{Methods}

In this article we solve the task of optimizing a HF wave energy source based on a numerical model. For this purpose some approved methods of the optimal control theory need an adaptation. The mathematical model which adequately reflects the process of the electron-wave energy exchange enables us to take the search for optimal functional dependencies down to solving the corresponding tasks of the optimal control.

Let's proceed from the equation for the movement of a charged particle in an electromagnetic wave:

$$
m \frac{d^{2} r}{d t^{2}}=q\{\mathbf{E}+[\mathbf{v} ; \mathbf{B}]\}
$$

and consider the following suppositions:

1. The movement of electrons is one-dimensional - along the axe of the device.

2. The powers of space charge are not taken into account.

3. Well-phased electron clots get to the entrance of the autophase section.

Let's also consider the movement of electrons in the field of the running high-frequency wave:

$$
E_{h f}=E_{h f}(z) \sin \varphi .
$$

The particle phase is taken as the wave phase, where the particle is placed at the time:

$$
\varphi=\omega \int_{0}^{z} \frac{d z}{d v_{p}(z)}-\omega t+\varphi_{0} .
$$

Given the fact that the electromagnetic field is axial-symmetric, we should multiply both parts of the equation (1) scalar by $\mathbf{v}$ :

Considering that

$$
\mathbf{v} m \frac{d^{2} r}{d t^{2}}=q\left\{\dot{z} E_{z}+\dot{r} E_{r}\right\} .
$$

$$
\mathbf{v} m \frac{d^{2} r}{d t^{2}}=\frac{d W}{d t}
$$

and restricting ourselves to the analysis of the movement of paraxial electrons from (4) we get an equation for determining the full energy of the clot $W$ in dependence from the distance it has passed along the autophase section (axe $z$ ):

$$
\frac{d W}{d z}=q E_{h f}(z) \sin \varphi+q E_{s t} .
$$

Another equation that illustrates the change of the phase along the axe $z$ can be derived by differentiation by $z$ :

$$
\frac{d \varphi}{d z}=\omega\left(\frac{1}{v_{p}}-\frac{1}{v_{z}}\right),
$$

where $v_{z}$ is the longitudinal speed of the particle. Equations (6) and (7) that describe the longitudinal motion of the clot depict quite well the real movement when the activity of the 
clot is low. However, the analysis of the dynamics of the longitudinal movement with the help of analytical methods can be applied not everywhere. The analytical methods can be used only when $E_{h f}(z)=$ const and $E_{s t}(z)=$ const. In other cases the particles movement is to be analyzed with the help of numerical methods. Given this, the following problem can be set with reference to the longitudinal movement.

Assume that the following quantities are fixed: the length of the autophase section, particle capture factor, the average particle speed at the entrance to the autophase section. The task is to define the amplitude change law of the high-frequency field and the strength change law of the static field in order to determine the minimum width of the phase spectrum und the minimum dispersion of the speed at the maximum energy exchange. This would mean that the clot is kept in the capture mood and the energy transmission is unilateral.

In order to solve this task it would be sufficient to analyze the phase field at the width of $2 \pi$ due to the periodical nature of the high-frequency field. When $\varphi \in[-\pi, 0]$ the clot will be set in the accelerating half-wave (reverse energy transformation). When $\varphi \in[0, \pi]$ the clot will be set in the inhibiting half-wave (direct energy transformation).

Let's introduce new variables to the equations (6) and (7):

$$
\begin{aligned}
& \frac{d \varphi}{d s}=2 \pi\left(\frac{1}{\beta_{p}}-\frac{1}{\sqrt{2 \varepsilon}}\right), \\
& \frac{d \varepsilon}{d s}=a(s) \sin \varphi+b(s) .
\end{aligned}
$$

Functions $a(s), b(s)$, that correspondingly determine the amplitude change law of the highfrequency field and the strength change law of the static field, will be further called as managements and denoted as $f_{1}=a(s), f_{2}=b(s)$.

Given this, (8), (9) will be transformed to:

$$
\begin{aligned}
& \frac{d \varphi}{d s}=2 \pi\left(\frac{1}{\beta_{p}}-\frac{1}{\sqrt{2 \varepsilon}}\right), \\
& \frac{d \varepsilon}{d s}=f_{1}(s) \sin \varphi+f_{2}(s) .
\end{aligned}
$$

It is only natural to analyze the managements $f_{1}$ and $f_{2}$ in the group of partially differentiated functions with restrictions

$$
\begin{aligned}
& f_{1 \text { min }} \leq f_{1}(s) \leq f_{1 \text { max }}, \quad f_{2 \text { min }} \leq f_{2}(s) \leq f_{2 \max }, \\
& \frac{d f_{1}(s)}{d s} \leq d_{11}, \quad \frac{d f_{2}(s)}{d s} \leq d_{12},
\end{aligned}
$$

that determine the possibilities of their practical realization.

Here $f_{1 \text { min }}, f_{1 \text { max }}, f_{2 \min }, f_{2 \max }, d_{11}, d_{12}$ are set constants. We should also assume that the equations can only have simple discontinuities which means the existence of final limits

$$
\begin{array}{rr}
f_{1}(\tau-0)=\lim _{s>\tau}^{s \rightarrow \tau} f_{1}(s), & f_{1}(\tau+0)=\lim _{s<\tau}^{s \rightarrow \tau} f_{1}(s), \\
f_{2}(\tau-0)=\lim _{s>\tau}^{s \rightarrow \tau} f_{2}(s), & f_{2}(\tau+0)=\lim _{s<\tau}^{s \rightarrow \tau} f_{2}(s),
\end{array}
$$

at discontinuity points there exist inequalities 


$$
\begin{aligned}
& \left|f_{1}(\tau+0)-f_{1}(\tau-0)\right| \leq d_{21} \\
& \left|f_{2}(\tau+0)-f_{2}(\tau-0)\right| \leq d_{22}
\end{aligned}
$$

where $d_{21}, d_{22}$ are constants.

Let's assume that $\bar{M}_{0}=\bar{M}_{0}\left(\varepsilon_{0}, \varphi_{0}\right)$ is a limited closed multitude of initial phases and particle energies. Any particle capture with the initial phase value of $\varphi_{0}$ at the entrance to the autophase section means that given, $s \in[0, L]$, where $L$ is the length of the autophase section, the particle phase change should be set as following:

for the direct energy transformation.

$$
0 \leq \varphi(s) \leq \pi
$$

Now let's introduce the functional:

$$
I\left(f_{1}, f_{2}\right)=\int_{M_{L}, f_{1}, f_{2}} g_{1}\left(\varepsilon_{L}, \varphi_{L}\right) d \varepsilon_{L}, d \varphi_{L} .
$$

Phase restrictions can be taken into account if we introduce an additional item to the functional:

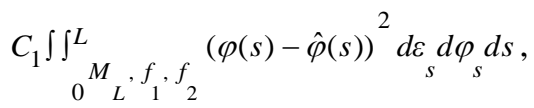

where $C_{1}$ is a weight constant and $\hat{\varphi}(s)$ is a middle phase. All limitations for derivatives of governing functions can be taken into account if we limit them to phase restrictions be introducing new phase variables.

Let's optimize the longitudinal movement of the clot based on the gradient methodic and apply it to the functional (15). In this case we will use the direction of the lowering, which can be established based on characteristic particles. The particle dynamics is depicted in the equation (9). Let's analyze the functional

$$
I_{1}\left(f_{1}, f_{2}\right)=\int_{M_{L}, f_{1}, f}\left\{A\left(\varepsilon_{L}-\bar{\varepsilon}(L)\right)^{2}+B\left(\varphi_{L}-\bar{\varphi}(L)\right)^{2}\right\} d \varepsilon_{L}, d \varphi_{L},
$$

where $A, B$ are weight constants, that are being introduced for practical reasons. The multitude $\bar{M}_{0}=\bar{M}_{0}\left(\varepsilon_{0}, \varphi_{0}\right)$ is set as a rectangle

$$
M_{0}=\left\{\left(\varepsilon_{0}, \varphi_{0}\right): \varepsilon_{0} \in\left[\varepsilon_{\min }, \varepsilon_{\max }\right], \varphi_{0} \in\left[\varphi_{\min }, \varphi_{\max }\right]\right\} .
$$

Next task is to minimize the functional (17). The direction of the lowering (anti-gradient) can be set as

$$
\bar{q}\left(s, f_{1}, f_{2}\right)=\left(q_{f_{1}}(s), q_{f_{2}}(s)\right)
$$

where

$$
\begin{aligned}
& q_{f_{1}}(s)=\int_{\bar{M}_{0}} \psi_{1}(s)(\sin \varphi(s)+1) d \varphi_{0} d \varepsilon_{0}, \\
& q_{f_{2}}(s)=2 \pi \int_{\bar{M}_{0}} \psi_{2}(s) d \varphi_{0} d \varepsilon_{0} .
\end{aligned}
$$

$\psi_{1}(s)$ and $\psi_{2}(s)$ are to be set from the system

$$
\begin{aligned}
& \frac{d \psi_{1}}{d s}=-\frac{\pi}{(2 \varepsilon(s))^{3 / 2}} \psi_{2}, \\
& \frac{d \psi_{2}}{d s}=f_{1}(s) \cos \varphi(s) \psi_{1}
\end{aligned}
$$


and final conditions

$$
\begin{aligned}
& \psi_{1}(L)=-2 A(\varepsilon(L)-\bar{\varepsilon}(L)), \\
& \psi_{2}(L)=-2 B(\varphi(L)-\bar{\varphi}(L)) .
\end{aligned}
$$

We divide rectangle (18) (multitude $\bar{M}_{0}$ ) along the axe $\varphi$ by $N$ equal parts $l_{i}$, $i=1,2, \ldots N$ and introduce integrals (19) and (20) as the following sums:

$$
\begin{gathered}
q_{f_{1}}(s)=h \sum_{i=1}^{N} \psi_{1}\left(s, \varepsilon_{0}, \varphi_{0 i}\right)\left(\sin \varphi\left(s, \varepsilon_{0}, \varphi_{0 i}\right)+1,\right. \\
q_{f_{2}}(s)=h \sum_{i=1}^{N} \psi_{1}\left(s, \varepsilon_{0}, \varphi_{0 i}\right),
\end{gathered}
$$

where $h=\operatorname{mes}\left(l_{i}\right), \varphi_{0 i} \in l_{i}$.

Similarly we get the following presentation of the functional

$$
I_{1}\left(f_{1}, f_{2}\right)=h \sum_{i=1}^{N}\left\{A\left(\varepsilon_{L i}-\bar{\varepsilon}(L)\right)^{2}+B\left(\varphi_{L i}-\bar{\varphi}(L)\right)^{2}\right\},
$$

where

$$
\begin{gathered}
\varepsilon_{L i}=\left(L, \varepsilon_{0}, \varphi_{0 i}, f_{1}, f_{2}\right), \\
\varphi_{L i}=\varepsilon\left(L, \varepsilon_{0}, \varphi_{0 i}, f_{1}, f_{2}\right), \\
\bar{\varphi}(L)=\frac{1}{N} \sum_{i=1}^{N} \varphi_{L i}, \\
\bar{\varepsilon}(L)=\frac{1}{N} \sum_{i=1}^{N} \varepsilon_{L i}
\end{gathered}
$$

Thus, we can establish the following algorithm of the search for stationary managements:

1. Determine the initial managements $f_{1}^{(0)}(s) ; f_{2}^{(0)}(s)$. Remember them in $k+1$ points of the interval $[0, L]$, carry out the spline-interpolation between the points.

2. Integrate $N$ times from 0 to $L$ system (10), given the managements $f_{1}^{(0)}(s) ; f_{2}^{(0)}(s)$ with the initial data $\varepsilon(0)=\varepsilon_{0 i}$ and $\varphi(0)=\varphi_{0 i}$ and calculate the functional (25). Remember the exit values $\varepsilon_{L i}, \varphi_{L i}, i=1,2, \ldots N$ and determine the average phase $\bar{\varphi}(L)$.

3. Integrate $N$ times in reverse order from $L$ to 0 system (21), given conditions (22) in parallel with system (10). Simultaneously carry out summations (23), (24), this way we determine the lowering direction $q\left(s, f_{1}^{(0)}, f_{2}^{(0)}\right)$.

4. Determine new managements and calculate restrictions (11) and (12). The step along the anti-gradient $\Delta \sigma$ should be chosen with regard to the minimum of the functional (25) in the given direction. Managements $f_{1}^{(1)}(s), f_{2}^{(1)}(s)$, that have been found after the lowering, should be taken as initial. Carry out procedures 1-4 one more time.

\section{Results}

The numerical optimization has been carried out for 24 particles in the autophase section with the following parameters: the length of the high-frequency wave $-3 \mathrm{~cm}$, the length of the autophase section $-14 \mathrm{~cm}$, the capture coefficient $-0,78$. The managements $f_{1}=a(s)$ 
and $f_{2}=b(s)$ have been determined by 30 points (with the interval of $0,5 \mathrm{~cm}$ between them), interpolation between them has also been carried out. Restrictions to the governing functions have been introduced given the fact, that the tensity of the high-frequency field will not change more then by $10^{6} \mathrm{~V} \backslash \mathrm{m}$, the tensity of the static field - not more then by $10^{4}$ $\mathrm{V} / \mathrm{m}$.

Given the fact, that an efficient energy exchange between the field of the electromagnetic wave and the static field corresponds to the finite movement of the clot and the wave near the local minimum of the potential energy, it is necessary to put some restrictions on the change of the static and high-frequency fields. This restrictions are practically steadiness conditions for the oscillators` movement [6]:

$$
\frac{\left|E_{s t}\right|}{E_{h f}}<\frac{|q| \omega^{2} R_{c r}}{\left|v_{0}-f_{0}\right|}
$$

where $q$ is the charge of a large particle, $R_{c r}$ - connection resistance, $f_{0}$ - the group speed that corresponds to the phase speed.

It is convenient to search for the optimal change law given $E_{h f}(z)$ and $E_{s t}(z)$ in the group of monotonously changing functions. The optimization has been carried out with regard to different restrictions for maximum values $f_{1}=a(s)$ and $f_{2}=b(s)$, also with regard to different values of the static and high-frequency fields given condition (26).

The values of the managements, that have been established as a result of the optimization of the autophase section by given restrictions are represented at Fig. 1. The initial managements correspond to the linear law of the static field $E_{s t}(z)$ change law, that is mostly arbitrary and is being restricted only by the steadiness of the clot movement (26).

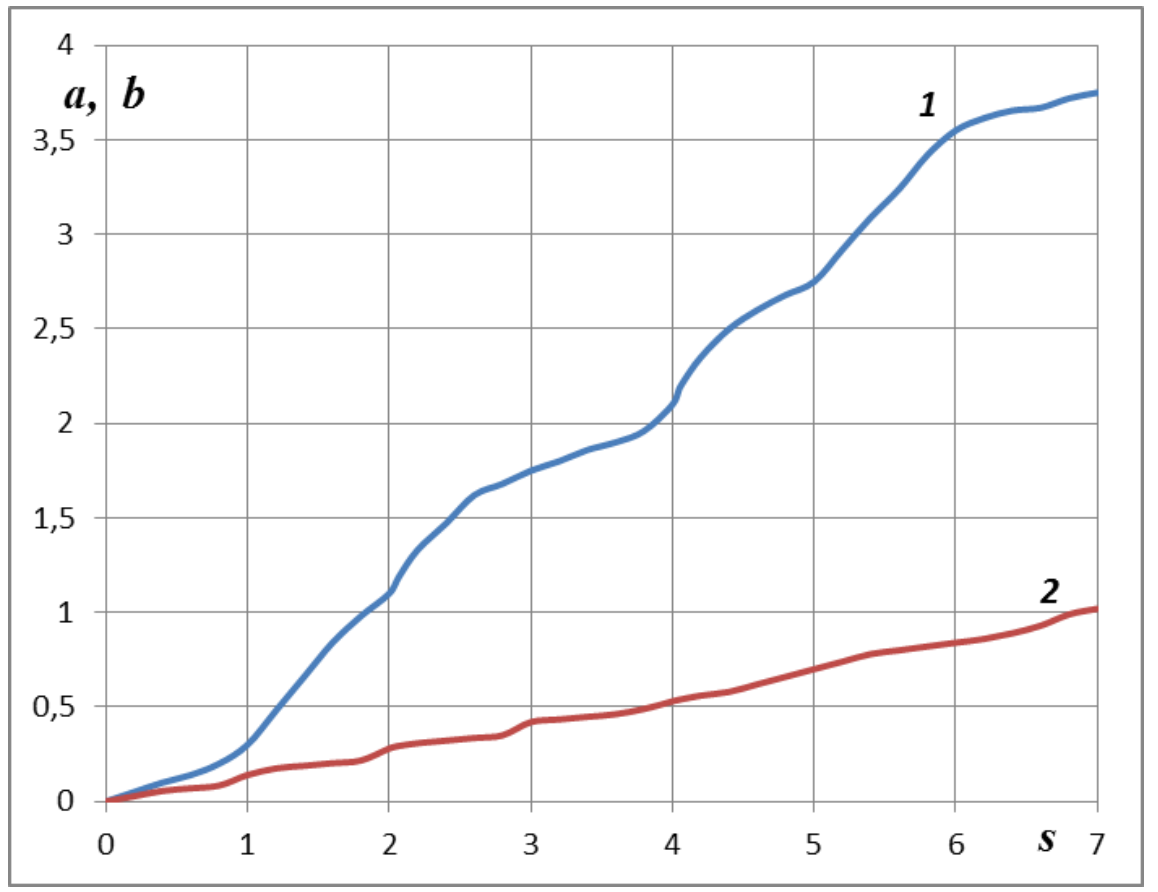

Fig. 1. Optimized managements: $1-a(s) ; 2-b(s)$. 
The lowering down the characteristic particles that mostly influence the value of the functional (25) has started form the initial managements. A computer realization of the given algorithm shows almost stationary managements. It is not necessary to determine the exact minimum (25). It is only important that the functional decrease is considerable. The established managements considerably improve the energy exchange that goes simultaneously with the diminution of the phase width of the clot. Functional (25) value hase decreased from 9,75 to 0,32 .

\section{Discussions}

Established managements improve the energy exchange twice as much, at the same time the phase width of the clot changes lightly, which corresponds to the fact that the greater part of the particles are being kept in the capture mood. By choosing coefficients A and B in (25) appropriately, lower phase dispersal at the exit of the autophase section can be achieved by maximum energy exchange. The value of the functional will decrease from 15,92 to 1,86 . Another profile of the static field tensity is possible by changing the initial conditions and restrictions to the managing functions. This profile will be more optimal from the point of view of the energy exchange, but it will also demand a different technical solution for the device.

Thus, the given and settled task of the optimal management for the energy exchange process between an electron clot and a high-frequency wave enables us to find optimal dependencies (managements) that describe the laws of tensity change for electrostatic and high-frequency fields. Conducted calculations show that the established functional dependences help keep the clot on the capture mood longer and stable, as well as enhance the efficiency of the energy exchange. In that way, using HF wave electronic devices with an autophase section in dielectric heating plants turns out to be quite advisable.

\section{References}

1. E. Jerby , Y. Meir Y, Ampere 2015-15th international conference on microwave and high frequency heating, 35 (2015)

2. W. Li, J. Peng, X. Wang, Environmental Technology, 35, 532 (2014)

3. A. Morozov, O. Morozov, V. Trebukh, D. Kalimullin, A. Prokopenko, Actual problems of electronic instrumentation of APEP-2016, 787 (2017)

4. A. Gerasev, Energy, 119, 989 (2017)

5. F. Chiadini, A. Scaglione, A. Diovisalvi, V. Fiumara, J. of Applied Physics, 116, 503 (2014)

6. I. Vorotyntseva, V. Kaschintseva, Scientific and Technical Volga region bulletin, 6, 145 (2017) 